kes seines Handbuches nicht ganz nötig. Ähnlich wie niemand den ganzen Wortschatz einer Sprache beherrscht, so operiert auch selten jemand aktiv mit allen grammatischen Strukturen. Für die aktive Beherrschung einer Sprache genügt die Beherrschung einer bestimmten Auswahl der Haupt- oder Grundstrukturen. Die anderen braucht man sich nur passiv anzueignen. Es bleibt allerdings die Frage, welche Strukturen wichtig und welche entbehrlich sind. Aber schon heute darf man feststellen, dass die Struktur des einfachen Satzes grundsätzlicher als die des zusammengesetzten ist. Manche Satzarten kommen nur selten vor. Es ist nicht notwendig diese weniger wichtigen Strukturen aktiv zu beherrschen. Anstatt dessen soll man die Grundstrukturen völlig mechanisieren. Solch ein Vorgehen ist möglich, da sich beinahe jede komplizierte Struktur metasprachlich in einfachere transformieren lässt ${ }^{8}$. Man muss hier also eine bestimmte Auswahl treffen und richtige Proportionen einhalten, entsprechend der Frequenz des Vorkommens einzelner Strukturen.
Ausserordentlich sorgfältig wurden die Übungen (es fehlen nur, wie oben erwähnt, phonetische) bearbeitet und zusammengestellt. Die Satztafeln sind ein wichtiger Mechanisierungsfaktor ${ }^{9}$ des neuen Materials. Ausserdem sind die Übungen auch verschiedenartig.

Zum Schluss soll noch bemerkt werden, dass der Verfasser in einigen grammatischen Interpretationen fehlgegangen ist, so z. B. (S. 51) bei der Erklärung der Rektion der Präpositionen, die mal den 3. mal den 4. Fall „regieren”. Die Bewegung bzw. Ruhelage wird hier nicht allein durch das Zeitwort sondern vor allem durch die jeweilige Beziehung beider mittels der Präposition verbundener Erscheinungen gekennzeichnet, z. B, „Er geht auf die

Zusammenfassend betonen wir, dass das Handbuch von A. Nikiel dennoch $\mathrm{zu}$ denen gehört, die Neues mit sich bringen Manche Schwächen lassen sich beseitigen andere sind von objektiven Schwierigkeiten abhängig. Man muss sich also vorläufig mit einem gewissen Kompromiss zufriedengeben.

FRANCISZEK GRUCZA

\title{
Wörterbuch der Deutschen Gegenwartssprache
}

Hrsg. v. Ruth Klappenbach u. Wolfgang Steinitz; Bearb.: Ruth Klappenbach u. H. Malige-Klappenbach; Lfg 1/2: A - annehmen, Lfg 3: annehmlich-Aufbruch, Lfg 4 aubruch(s) - Ausführung; Berlin: Akademie Verlag, Lfg 1/2,3 - 1961; Lfg 4 - 1962 im Rahmen der Veröffentlichungen des Instituts für deutsche Sprache und Literatur der Deutschen Akademie der Wissenschaften zu Berlin

Das uns bereits in vier Lieferungen vorlegende Wörterbuch der deutschen Gegenwartssprache bedarf keiner besondren Reklame Es ist eben ein Wörterbuch nach dem seit längerer Zeit eine besondre Nachfrage seitens der Deutschen und der Ausländer, sei- tens der Lehrer und der Lernenden groß war. Auf Grund der bis jetzt erschienenen Lieferungen kann man schon heutzutage feststellen, daß die Wünsche all derer, die sich fur die deutsche Gegenwartssprache interessieren befriedigt und erfüllt werden. Es

8 Vgl. R. Jakobson Metajęzyk $w$ kręgu zagadnień lingwistycznych. In: Sprawozdania PTPN 20 I 1958, Nr 2, S. 157; L. Zabrocki. Sprachkode. In: Z. f. Phonetik, Sprachwissenschaft, Kommunikationsforschung, Bd. 14 (1961), H. 1, S. 64.

9 Die sprachliche Struktur wird mechanisiert, indem sich die sprachliche Form von dem konkreten Inhalt freilöst. Vgl. L. Zabrocki, Języki obce $w$ szkole. In: Nowa Szkoła, 1961, Nr. 4, S. 14 ff. 
ist ein Wörterbuch, das die gegenwärtige deutsche Sprache vom wissenschaftlichen Standpunkt aus betrachtet (zugleich in Hinsicht auf ihren recht verwickelten und umständlichen Charakter), dennoch aber genug klar, leicht und zugänglich für den Laien oder erst Lernenden, der im Wörterbuch einen Rat oder die Lösung eines Zweifels sucht. Es ermöglicht ebenfalls ein Nachschlagen der vielen Beispielssätze und Zitate, die für die einzelnen Stichwörter (einschließlich Werk und Seite) angegeben worden sind.

Mit großem Interesse verfolgen wir die bis jetzt erschienenen Lieferungen und schließen uns dem Wunsch vieler anderer an, daß weitere Lieferungen des Wörterbuches möglichst nicht in allzugroßen Zeitspannen weiter erscheinen.

Allgemeine Ziele des Wörterbuches werden im Vorwort erörtert. Es sind: „Das Wörterbuch der deutschen Gegenwartssprache hat die Aufgabe, den heutigen deutschen Wortschatz mit seinen Verwendungen darzustellen. Mit. dieser Aufgabe sind folgende Hauptziele verbunden: die Angabe der Bedeutung des Einzelwortes, seine stilistische Kennzeichnung, seine grammatische Kennzeichnung und seine Verwendung im Satz"1.

Das Wörterbuch erfreut sich außer der ihm freundlich zugeneigten Ansagen aus dem Jahre $1958^{2}$ einer genaueren Besprechung des geplanten Werkes von Dr. J. Scharnhorst ${ }^{3}$ und einer Rezension der ersten zwei Lieferungen von $H$. Moser aus dem Jahre $1961^{4}$.

Den Vorschlag, ein Wörterbuch der deutschen Gegenwartssprache herauszugeben, machte 1952 bei der Gründung des Instituts für deutsche Sprache und Literatur an der Deutschen Akademie der Wissenschaften zu Berlin, Prof. Dr. W. Steinitz. Seit dieser Zeit begann an dem Wörterbuch ein Kollektiv von mehreren Sprachwissenschaftlern zu arbeiten (für jede Lieferung werden die Autoren einzelner Artikel genannt), wobei die Hauptbearbeitung der bisher erschienenen Lieferungen in den Händen von R. Klappenbach und H. Malige-Klappenbach ruht.

Die Vorarbeiten zum Gesamtwerk sind riesengroß. Die Grundlage für die Bearbeitung einzelner Wortartikel bildet eine Wortkartei von über 700000 Zetteln. Das Wörterbuch soll ungefähr die Gesamtzahl von 50 bis 60 Lieferungen erreichen, also ungefähr 5 bis 6 Bände.

Ein besonders und keinesfalls leichtes Problem stellt für die Bearbeiter des neuen Wörterbuches die Wortwahl und die Wortbedeutung im heutigen Deutsch dar.

$\mathrm{Da}$ es für einen weiten Benutzerkreis bestimmt ist, und das nicht nur in Deutschland, sondern auch im Ausland, soll es vor allem „... die deutsche Sprache der bildungstragenden Schicht der Gegenwart darstellen. Unter der bildungstragenden Schicht sind die in Wissenschaft und Kunst, in Technik, Wirtschaft und Verwaltung, in den gesellschaftlichen Organisationen und Parteien verantwortlich tätigen Menschen verstanden, die die Sprache unseresöffentlichen Lebens sowie der schönen wissenschaftichen und technischen Literatur und Presse bestimmen" 5.

${ }^{1}$ R. Klappenbach u. W. Steinitz, - Vorwort zum Wörterbuch der deutschen Gegenwartssprache, S. 03, Lfg $1 / 2$.

${ }^{2}=$ Deutschunterricht, Volk u. Wissen, Volkseigener Verlag, Berlin, 3 (1958) S. 142-160; Muttersprache, Lüneburg, Heliand-Verlag, S. $196-98$ (1958).

${ }^{3}$ J. Scharnhorst, Das Wörterbuch der deutschen Gegenwartssprache, = Sprachpflege, 9. Jg. H. 12 (1960) S. $243-244$.

${ }^{4}$ H. Moser, Wörterbuch der deutschen Gegenwartssprache, Lfg. 1/2: A-annehmen, = Deutsche Literaturzeitung 82. Jg. H. 12 (1961) Sp. 1096-1100.

5 R. Klappenbach u. W. Steinitz, Vorwort zum Wörterbuch der deutschen Gegenwartssprache, S. 03, Lfg. $1 / 2$. 
Die Sprache der bildungstragenden Schicht wir ja meistens gesprochen und geschrieben, sie wird ja allen anderen Schichten auf verschiedene Art und Weise beigebracht, und unter ihnen verbreitet; somit hat man auch das "Allgemeinverbreitete, das Typische" ihres Wortschatzes sorgfältig erfaßt und womöglichst mit Beispielen und Belegen gesichert. Ausnahmen und Individuelles wird nicht berücksichtigt.

Eine weitgehende Einschränkung betrifft selbstverständlich ebenfalls die Aufnahme der Spezialtermine der Fachund Sondersprachen, wie auch der mundartlichen Ausdrücke, falls sie nicht allgemein bekannt sind.

Dafür werden verhältnismäßig viele Fremdwörter berücksichtigt. Sie sind in der heutigen deutschen Sprache tatsächlich reich vertreten, so daß ihre Rolle und Funktion im heutigen Deutsch in vielen Aufsätzen und Arbeiten einerseits kritisch, andrerseits aber auch (in mancher Hinsicht) positiv behandelt wird ${ }^{6}$.

Was verstehen die Autoren und Herausgeber des Wörterbuches unter der deutschen Gegenwartssprache? „Unter deutscher Gegenwartssprache wird außer so charakterisierten, heute geschriebenen und gesprochenen Sprache der bildungstragenden Schicht auch die Sprache unserer Zeit noch gelesenen, lebendigen deutschen Literatur der Vergangenheit verstanden. Daher fußt das Wörterbuch zwar vornehmlich auf dem Wortschatz des 20.Jh., zieht aber den der Literatur des 19.Jh. und in gewissem Umfang des letzten Drittel des 18. Jh. heran",

Es wird betont, daß das Wörterbuch vorwiegend ein Bedeutungswörterbuch ist. Wie hatte man daher die Bedeutungen der einzelnen Wortartikel bearbeitet? Eigentlich verzichten die Autoren auf etymologische und historische Deutungen. Die Angaben sind kurz und einfach; der entsprechende Begriff wird entweder umschrieben, es wird für ihn auch oft ein anderes, sinnverwandtes Wort oder ein Synonym angegeben, oder er erfährt auch gar keine Erklärung. Solche Artikel wie z.B. Auge, Nase $^{8}$, werden nur durch ihre stilistischen Verwendungsmöglichkeiten illustriert.

Es werden auch nur die wichtigsten grammatischen Angaben gemacht. Substantivierte Wortformen mit selbständigen Bedeutungen besitzen ihre eigenen Stichwörter, - wenn möglich werden sie außerdem in den für sie typischen Wendungen gebracht, z.B. Aber, das; -s, - Einwand, Bedenken: wenn das A. nicht wäre!; viele Wenn und A.; ... Schwierigkeit: die Sache hat ihr A.; es ist ein (großes) A. dabei, ... ${ }^{9}$

Interessant sieht die stilistische Bewertung des deutschen Wortschatzes aus. Im Zusammenhang mit dem oben erwähnten, hatte man den Gebrauch des Wortschatzes festgestellt, ihn entsprechend bewertet und charakterisiert; danach die einzelnen Wörter nach ihrer Lage in den bestimmten Wortfeldern verglichen und in die entsprechenden „Stilsphären” zugeordnet.

${ }^{6}$ Georg Möller, Deutsch von heute, Verlag der Enzyklopädie, Leipzig 1960; vor allem die Kapitel „Das Fremdwort-kritisch betrachtet”, S. 29, „Das fremde Verb”, S. 79; und die Artikelreihe unter d. Gesamttitel „Gedanken über Entwicklungstendenzen in der Sprache der Gegenwart", = Deutschunterricht, Volk und Wissen, Volkseigener Verlag, Berlin, Jg. 15 (1962)

R. Klappenbach u. W. Steinitz = Vorwort zum Wörterbuch d. dt. Gegenwartssprache, S. $04, \mathrm{Lfg} .1 / 2$.

${ }^{8}$ R. Klappenbach, Das Wörterbuch der deutschen Sprache der Gegenwart, III. Die Bedeutungen, = Deutschunterricht, 3 (1958) S. 144; vgl. außerdem die genauen Angaben über den Aufbau der Artikel und über ihre Bedeutungen im Vorwort zur Lfg. 1/2 des zitierten Wörterbuches.

${ }^{9}$ Wörterbuch der deutschen Gegenwartssprache, Lfg. 1/2, S. 14. 
Schließlich wurden auf Grund dieser Verfahrungsweise bei der Bewertung und stilistischer Charakterisierung des Wortschatzes vier Ebenen (in die der Wortschatz gegliedert werden kann) unterschieden: „A. die Stilsphären, $B$. die zeitliche Zuordnung, $C$. die räumliche Zuordnung, D. die fachliche Zuordnung” 10.

Die Kennzeichnung und Qualifizierung des Wortschatzes im Bereich der einzelnen Wortartikel und Stichwörter ist sehr eingehend und genau.

Das Wörterbuch enthält viel mehrere Zusammensetzungen als die älteren Wörterbücher anderer Verlage, was ja auch aus den Grundtendenzen und unbegrenzten Entwicklungsmöglichkeiten, wie auch der Aufnahme neuen Wortgutes, vor allem der Neuprägungen in die deutsche Gegenwartssprache $z u$ deuten ist ${ }^{11}$.

Auch an bestimmte Hinweise, die eine richtige deutsche Aussprache betreffen wurde gedacht, mit der Voraussetzung, daß die allgemeinen Ausspracheregeln dem Benutzer bekannt und geläufig sind ${ }^{12}$.

Man könnte im Zusammenhang damit wohl aber doch einige Vorbehalte haben, z.B. ob es wirklich für richtig gehalten werden kann, nicht die internationale Lautschrift in der Umschrift anzuwenden (trotzdem das Wörterbuch auch für das Ausland bestimmt ist), „sondern eine Umschrift, die soweit wie möglich mit der deutschen Orthographie auskommt", - also statt [ $[$, ts, v] die Umschreibung mit [sch, z, w] u.a. Man begründet es zwar damit, die gewählte Umschriftsform wäre lesbarer, und ,bei dem Ausländer, der das Wörterbuch benutzt, muß soviel Kenntnis des Deutschen vorausgesetzt werden, daß auch ihm keine Schwierigkeiten daraus erwachsen ${ }^{13}$. Trotzdem hatte man dennoch einige Zeichen aus der internationalen Lautschrift übernehmen müssen, wie $[3$ in: Journal, Garage, $[\mathrm{H}]$ in: singen, [0] in: all right, oder das Nasalierungszeichen [ ] in: Chance, Fond, Parfüm, Bassin [ã, õ, ü, ë]. Also - wenn schon, so konnte man ganz konsequent verfahren haben, um vielleicht dem doch nicht immer ganz gut phonetisch geschulten deutschlernenden Ausländer die einwandfreie Aussprachehilfe zu bieten.

Für die Slawen wäre z.B. die Unterscheidung des Ach- $[\chi]$ und des Ichlautes[ç] sehr angebracht (vor allem im Falle einer weichen Aussprache, z.B. Licht, oder in Fremdwörtern Technik, Mechanik. Da aber das Wörterbuch selbstverständlich nicht allen Wünschen nachgehen kann, hat der Benutzer im Wörterbuch aufmerksam das Vorwort zu studieren, ebenfalls die typographischen Erläuterungen und das Abkürzungsverzeichnis, das auf den Umschlagseiten zur Lieferung 3 geboten wird.

Hugo Moser äußert in seiner Rezension zur Lfg $1 / 2$ den Wunsch, bestimmte Wortartikel könnten genauer präzisiert werden und weist auf einige fehlenden Neuerungen (im östlichen Deutschland) ${ }^{14}$.

Wir möchten dagegen (als Ausländer) gerne betonen, daß trotz der vielen Unterschiede, die sich im Wortschatz (vor allem in den Neuwörtern, Neuprägungen und Neubedeutungen) der zwei deutschen Staaten nach 1945 beobachten und aufnehmen ließen, und trotzdem, daß ja im genannten Wörterbuch

${ }^{10} \mathrm{vgl}$ die frühere Einteilung in 5 Ebenen, $=\mathrm{K}$. Klappenbach, Das Wörterbuch d. dt. Sprache d. Gegenwart IV. Die Bewertungen (Deutschunterricht 3 (1958) S. 146 und die aktuelle/Vorwort zum Wörterbuch der dt. Gegenwartssprache, Lfg. 1/2, S. 010.

${ }^{11}$ Vgl. Vorwort zur Lfg. 1/2, S. 015, IV. Auswahl und Ansatz der Stichwörter.

12 Vgl. Vorwort zur Lfg. 1/2, S. 022, VII. Die Aussprache.

${ }^{13}$ Vorwort, Lfg. 1/2, VII. Die Aussprache, S. 023.

${ }^{14}$ H. Moser, Wörterbuch der dertschen Gegenwartssprache, Lfg. 1/2: A-annehmen, Deutsche Literaturzeitumg, 82. Jg. H. 12 (1961) Sp. 1099.

${ }^{15}$ Für das Schriftbild wurde der Leipziger Duden 1959 berücksichtigt. 
vor allem die gegenwärtige deutsche Sprache der DDR aufgenommen wird, das Bestreben nicht unterlassen wurde, möglichst gleichmäßig auch das Neue in der DBR $\mathrm{zu}$ berücksichtigen. Das allein sehen wir u.a. schon in der Kennzeichnung DDR und DBR in bezug auf die Unterschiede im Sprachgebrauch nach 1945.

\section{Duden-Ost}

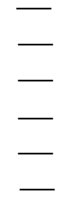

Arbeitskollege

Arbeitsproduktivität

Arbeiterstudent

Wortbedeutung

AGITATION:

1. Werbung

2. Aufklärung über aktuelle Tagesfragen

3. eine Tätigkeit gerichtet auf die Verbreitung politischer Ideen

AGGRESSION:

1. widerrechtlicher Angriff Angriff, Überfall

2. imperialistischer Überfall

AKTIV:

1. Tätigkeitsform der Zeitwörter

2 Arbeitsgruppe, die kollektiv an der Erfüllung gesellschaftspolitischer wirtschaftlicher u. kultureller Aufgaben arbeitet und überdurchschnittliche Leistungen anstrebt

AKTIVIST:

1. zielbewußt Handelnder, sehr aktiver Mensch

2. anerkannter Neuerer im Arbeitsprozeß

Gegenwartssprache

Arbeitskollege

Arbeitsfeld

klärungstätigkeit
Es lassen sich natürlich verschiedene Unterschiede in der Wortverwendung und in der Interpretation der Bedeutung notieren. Wir möchten es an einigen charakteristischen Beispielen (verglichen mit der Leipziger Duden Ausgabe 1959 und der Mannheimer Duden-Ausgabe 1958) illustrieren:

W o r t v e r w e n d u n g

Wörterbuch der deutschen

Arbeitsproduktivität

Arbeitslosenversicherung

politische Werbearbeit, Auf-

Duden-West

Amtsgerichtsrat

Amtstracht

Arbeitgeber

arbeitlich

Arbeitnehmer

Arbeitskamerad

Arbeitsfeld

Arbeitslosenversicherung

1. Werbung

2. politische

Aufreizung

rechtwidriger

Angriff

Sprachlehre-

Tatform

2. Neubedeut. DDR

Arbeitsgruppe zur Beratung $u$. Erfüllung bes. Aufgaben: ein pädagogisches, technisches A.

aktiver Mensch, zielbewußt zielbewußt und energisch Handelnder; Handelnder Neubedeut. DDR Werktätiger, der vorbildliche Leistungen vollbracht hat (und dafür ausgezeichnet worden ist) 
Neuprägungen-DDR:

Arbeiterkolonie-Siedlung für Fabrikarbeiter; Arbeiterkontrolle-Kontrolle durch die Arbeiter; Arbeiterstudent-Student, der vor seinem Studium Arbeiter war; $A r$ beiter- und- Bauern-Fakultät (Abk.: ABF)-der Universität angegeliederte Bildungsanstalt für Arbeiter und Bauern zur Erwerbung der Hochschulreife; $A r$ beitsbrigade- eine A. bilden, mehrere Arbeitsbrigaden aufstellen, der Wettbewerb der A. untereinander; Arbeitseinheit- (Einheitsmaß für Arbeitsleistung) die (menschliche) Arbeit nicht nach Zeit, sondern nach A. berechnen; ... Arbeitsgrundlage; Arbeitskollektiv; Arbeitskränelenkung-behördliche Lenkung der Arbeitskräfte; Arbeitsnorm-Norm der in einer bestimmten Zeit zu leistenden Arbeit; Arbeitsnormung-Festsetzung der Zeit für die Ausführung einer bestimmten Arbeit; Arzt, verdienter A. des Volkes; Arzthelfer — medizinisch geschulte Kraft, die als Arzt eingesetzt, ist; Ärzte-:(beratungs) kommission-Kommission von Ärzten, die über die Arbeitsfähigkeit eines Patienten berät, Aspirant, der;-en,-en - wissenschaftliche Nachwuchskraft, die sich besonders ihrer Weiterbildung widmen darf; $A s$ pirantur; die;-,-en - besonderer Ausbildungsgang für den wissenschaftlichen Nachwuchs; Attestation, die;-,-en - Ertei- lung der Lehrbefähigung unter Erlaß gewisser Prüfungen; Aufbauhelfer, der; Aufklärungsaufsatz, - Agitationseinsatz; Aufklärungslokal-ein der Agitation dienender öffentlicher Raum; aufschlüsseln-(Neubedeutung)-einen Plan auf die einzelnen Betriebe, Abteilungen, Brigaden a. (die Planaufgaben den einzelnen Betrieben, Abteilungen, Brigaden zuordnen) $\mathrm{N}$ e u b e d e u t u n g-B R D :

Arbeitsfriede(n), ungestörter Verlauf der Arbeit; a) Ruhe bei der Arbeit: b) Neubedeutung BRD-Verhältnis zwischen Arbeitgeber u. Arbeitnehmer, in dem Kampfmaßnahmen unterbleiben: den A. stören, wiederherstellen; Arbeitsteam.; Aufbaudarlehen;

Im allgemeinen ist das Wörterbuch der deutschen Gegenwartssprache eine sehr gute Probe dessen, wie man die Wissenschaftlichkeit mit der Allgemeinverständlichkeit gut vereinbaren kann. Als besonders interessant finden wir die genaue und zugleich höchst objektive Beurteilung, Unterscheidung und Qualifizierung der Neubedeutungen, Neuprägungen und der Neuwörter.

Das Wörterbuch der deutschen Gegenwartssprache ist ein Beispiel dafür, wie die Wörterbücher der gegenwärtigen Sprachen überhaupt zu bearbeiten sind.

K. TOBY-TERESZYŃSKA

St. Schimitzek, B. Sypniewska, M. Zurakowska: Kleinwörterbuch, deutsch-polnisch. Warszawa 1962 XX + 389 Seiten

Das Interesse für Fremdsprachen in Polen wirkt sich auch auf dem Gebiete der Lexikographie aus, leider nur sehr langsam und noch immer in einem den Erfordernissen nicht entsprechenden Umfang.

Das vorliegende Wörterbuch (mit etwa 12000 Stichwörtern) gehört zu den nennenswerten deutsch-polnischen Wörterbüchern, die in der Nachkriegszeit erschienen sind*. Es ist vor allem für den Touristen bestimmt und bringt unter diesem Gesichtspunkt eine Auswahl von Wörtern, die dem Bereiche des Fremdenverkehrs, des Alltagslebens, Sport u.a. entnommen sind. Begrüßenswert sind die Bemühungen der Verfasser, auch modernes Wortgut aufzunehmen (Düsenflugzeug, Trennschärfe, atomgetrieben, atomwaffenfrei u.a.).

Der Aufbau der Artikel ist übersichtlich und klar, den knappen grammatischen Angaben folgen die polnischen Entsprechungen. Wo das Stich- 